\title{
Cross Z-Complementary Pairs (CZCPs) for Optimal Training in Broadband Spatial Modulation Systems
}

\author{
Zilong Liu*, Ping Yang ${ }^{\dagger}$, Yong Liang Guan ${ }^{\ddagger}$, and Pei Xiao ${ }^{\S}$ \\ ${ }^{*}$ University of Essex, UK (E-mail: zilong. liueessex.ac.uk). \\ ${ }^{\dagger}$ University of Electronic Science and Technology of China (E-mail: yang.ping@uestc.edu.cn). \\ ${ }_{\ddagger}^{\ddagger}$ Nanyang Technological University, Singapore (E-mail: eylguan@ntu.edu.sg). \\ ${ }^{\S}$ University of Surrey, UK (E-mail: p.xiao@surrey.ac.uk).
}

\begin{abstract}
Spatial modulation (SM) is a new multiple-input multiple-output (MIMO) paradigm in which only one transmit antenna is activated over every symbol duration. So far, efficient SM training sequences (different from the existing design for conventional MIMO systems) remain largely open. Motivated by this research problem, we introduce a novel class of sequence pairs, called "cross Z-complementary pairs (CZCPs)", each displaying zero-correlation zone (ZCZ) properties for both their aperiodic autocorrelation sums and crosscorrelation sums. A CZCP may be transmitted in two non-orthogonal SM channels and hence proper design should be conducted to minimize the cross-interference of the two constituent sequences. We construct perfect CZCPs based on selected Golay complementary pairs. We show that the training sequences derived from our proposed CZCPs lead to optimal channel estimation performance over frequency-selective SM channels.
\end{abstract}

\section{INTRODUCTION}

This work ${ }^{1}$ presents a novel class of sequence pairs called "cross Z-complementary pairs (CZCPs)" and their applications for training sequence design in broadband spatial modulation (SM) systems. In what follows, we introduce the state-ofthe-art of SM and "pairs of sequences", followed by the contributions of this work.

\section{A. Spatial Modulation (SM)}

SM is a special class of MIMO techniques which trades multiplexing gain with complexity and performance [1]-[3]. Unlike conventional MIMO, an SM system is equipped with multiple transmit antenna (TA) elements but only a single radio-frequency (RF) chain. During each time-slot, an SM symbol can be divided into two parts: one (called "spatial symbol") is responsible for the selection and then activation of a TA element, the other (called "constellation symbol") is selected from a conventional PSK/QAM constellation and transmitted from the active TA element. Such unique transmission principle of SM allows it to have the salient advantages of zero inter-channel interference, low energy consumption, and low receiver complexity. Up to date, however, little has been understood on channel estimation of SM in frequency-selective channels. Early literature on SM mostly assume that channel state information (CSI) is perfectly known at the receiver [4], [5]. Effects of channel estimation errors to the performance of SM systems, limited to flat-fading channels, have been studied

\footnotetext{
${ }^{1}$ A full version of this work is given at: https://arxiv.org/abs/1909.10206.
}

in [6]-[9]. Note that the "one-RF-chain" principle of SM prevents the transmitter from using simultaneous pilot transmission over all the TAs. Consequently, it implies that dense training sequences for traditional MIMO [10] are unapplicable in SM systems. Although an identity training matrix has been employed for joint channel estimation and data detection in SM systems [8], extension to frequency-selective channels is not straightforward. A naive scheme is to extend a perfect sequence (having zero autocorrelation sidelobes) with cyclicprefix (CP) and then send the extended sequence in turn over multiple TAs. But this training scheme would be inefficient in highly dispersive channels.

\section{B. Pairs of Sequences}

Pairs of sequences play an important role in engineering particularly in wireless communications. A celebrated class of sequence pairs is called Golay complementary pairs (GCPs) which were proposed by Marcel J. E. Golay in 1951 [11] in his design of infrared multislit spectrometry. Let $\mathbf{a}$ and $\mathbf{b}$ be two sequences of identical length. Denote by $\rho(\mathbf{a}, \mathbf{b})(\tau)$ their aperiodic crosscorrelation (ACC) at time-shift $\tau$ (formal definition of ACC will be given in Section II). For simplicity, when $\mathbf{a}=\mathbf{b}, \rho(\mathbf{a}, \mathbf{b})(\tau)$ will be written as $\rho(\mathbf{a})(\tau)$. By definition, $(\mathbf{a}, \mathbf{b})$ is said to be a Golay complementary pair (GCP) if $\rho(\mathbf{a})(\tau)+\rho(\mathbf{b})(\tau)=0$ for all $\tau \neq 0$. In principle, sequences in a GCP work in a cooperative way so that their aperiodic autocorrelations (AAC) sums vanish for all the nonzero time-shifts [12].

Recursive algorithms proposed by Budišin in 1990s for polyphase and multi-level GCPs can be found in [13], [14]. Theory on para-unitary (PU) matrices has recently been applied for more efficient GCP synthesis [15]-[18]. In 1999, Davis and Jedwab developed a direct construction of polyphase GCPs through generalized Boolean functions [19]. Their construction was extended to GCPs over QAM constellations using weighted sum of several QPSK GCPs [20], [21]. Existing known binary GCPs have even lengths of the form $2^{\alpha} 10^{\beta} 26^{\gamma}$ only, where $\alpha, \beta, \gamma$ are non-negative integers satisfying $\alpha+\beta+\gamma \geq 1$ [22]. For sequence pairs with odd lengths, the best known alternative to GCPs is optimal odd-length binary Z-complementary pairs (OB-ZCPs) [23] each displaying the largest zero-correlation zone (ZCZ) width [24] and minimum AAC sums outside the ZCZ. 
It should be noted that 1) Each GCP or ZCP is defined by their AAC sums only; 2), Orthogonal channels are needed for the transmission of each sequence pair.

\section{Contributions of This Paper}

In this work, we introduce a novel class of sequence pairs, called $C Z C P s$, each displaying certain $\mathrm{ZCZ}$ properties for both their AAC sums and ACC sums. Specifically, a pair of sequences, say $(\mathbf{a}, \mathbf{b})$, is called a CZCP if $\rho(\mathbf{a})(\tau)+\rho(\mathbf{b})(\tau)$ and $\rho(\mathbf{a}, \mathbf{b})(\tau)+\rho(\mathbf{b}, \mathbf{a})(\tau)$ take zero values for certain timeshifts $\tau$. The most distinctive feature of CZCPs (compared to conventional sequence pairs) is that the two constituent sequences in a CZCP may interfere with each other during the transmission. Therefore, careful design should be carried out to force their cross-interference to zero in a certain $\tau$ region. We investigate the structural properties of CZCPs and show that a subset of GCPs are perfect CZCPs.

Then, we design optimal SM training sequences (which are equivalent to certain sparse matrices) with the aid of CZCPs. We provide numerical simulations to show that our proposed SM training sequences lead to minimum channel estimation mean-squared-error (MSE).

\section{PRELIMINARIES}

The following notations will be used throughout this paper.

- $\underline{\mathbf{x}}$ denotes the reversal of vector $\mathbf{x}=\left[x_{0}, x_{1}, \cdots, x_{N-1}\right]^{\mathrm{T}}$, i.e., $\underline{\mathbf{x}}=\left[x_{N-1}, x_{N-2}, \cdots, x_{0}\right]^{\mathrm{T}}$;

- $\mathbf{1}_{m \times n}$ and $\mathbf{0}_{m \times n}$ denote an all-1 matrix and an all-0 matrix, respectively, both having matrix order of $m \times n$;

- Denote $\omega_{q}=\exp \left(\frac{2 \pi \sqrt{-1}}{q}\right)$ (integer $q$ );

- $\mathbb{Z}_{q}$ denotes the set of integers modulo $q$, i.e., $\mathbb{Z}_{q}=$ $\{0,1, \cdots, q-1\}$ ( $q$ integer);

- $\mathcal{A}_{q}=\left\{\omega_{q}^{0}, \omega_{q}^{1}, \cdots, \omega_{q}^{q-1}\right\}$ denotes the set over $q$ complex roots of unity.

For two length- $N$ complex-valued sequences

$$
\mathbf{a}=\left[a_{0}, a_{1}, \cdots, a_{N-1}\right]^{\mathrm{T}}, \quad \mathbf{b}=\left[b_{0}, b_{1}, \cdots, b_{N-1}\right]^{\mathrm{T}},
$$

denote by $\rho(\mathbf{a}, \mathbf{b})(\tau)$ the ACC between $\mathbf{a}$ and $\mathbf{b}$, i.e.,

$$
\rho(\mathbf{a}, \mathbf{b})(\tau)= \begin{cases}\sum_{n=0}^{N-\tau-1} a_{n} b_{n+\tau}^{*}, & 0 \leq \tau \leq N-1, \\ \sum_{n=0}^{N+\tau-1} a_{n-\tau} b_{n}^{*}, & -(N-1) \leq \tau \leq-1, \\ 0, & |\tau| \geq N .\end{cases}
$$

In particular, when $\mathbf{a}=\mathbf{b}, \rho(\mathbf{a}, \mathbf{a})(\tau)$ will be sometimes written as $\rho(\mathbf{a})(\tau)$ and called the AAC of a at time-shift $\tau$.

Definition 1: $(\mathbf{a}, \mathbf{b})$ is called a GCP if their AAC sum equals zero for any non-zero time-shift $\tau$, i.e.,

$$
\rho(\mathbf{a})(\tau)+\rho(\mathbf{b})(\tau)=0, \forall \tau \neq 0 .
$$

Furthermore, two GCPs $(\mathbf{a}, \mathbf{b})$ and $(\mathbf{c}, \mathbf{d})$ are said to be mutually orthogonal if they have zero ACC sums for all timeshifts, i.e.,

$$
\rho(\mathbf{a}, \mathbf{c})(\tau)+\rho(\mathbf{b}, \mathbf{d})(\tau)=0,
$$

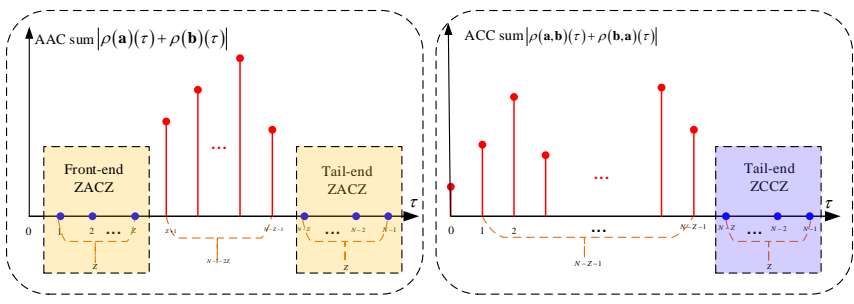

Fig. 1: Illustrative plots for the correlation properties of $(N, Z)$-CZCP

holds for any arbitrary $\tau$. As a matter of fact, $\left(\underline{\mathbf{b}^{*}},-\underline{\mathbf{a}}^{*}\right)$ is a GCP which is mutually orthogonal to $(\mathbf{a}, \mathbf{b})$ [22], where $\underline{\mathbf{b}}$ denotes the reversal of $\mathbf{b}$.

\section{Cross Z-Complementary PAIRs: Properties AND CONSTRUCTIONS}

This section studies the properties and constructions of CZCPs. Throughout this paper, we focus on polyphase $q$-ary CZCPs whose entries are drawn from $\mathcal{A}_{q}$. Before proceeding further, we formally define CZCPs as follows.

Definition 2: Let $(\mathbf{a}, \mathbf{b})$ be a pair of sequences of identical length $N$. For a proper integer $Z$, define $\mathcal{T}_{1} \triangleq\{1,2, \cdots, Z\}$ and $\mathcal{T}_{2} \triangleq\{N-Z, N-Z+1, \cdots, N-1\}$. $(\mathbf{a}, \mathbf{b})$ is called an $(N, Z)$-CZCP if it possesses symmetric zero (out-of-phase) AAC sums for time-shifts over $\mathcal{T}_{1} \cup \mathcal{T}_{2}$ and zero ACC sums for time-shifts over $\mathcal{T}_{2}$. In short, an $(N, Z)$-CZCP needs to satisfy the following two conditions.

$$
\begin{aligned}
& \mathrm{C} 1: \rho(\mathbf{a})(\tau)+\rho(\mathbf{b})(\tau)=0, \text { for all }|\tau| \in \mathcal{T}_{1} \cup \mathcal{T}_{2} ; \\
& \mathrm{C} 2: \rho(\mathbf{a}, \mathbf{b})(\tau)+\rho(\mathbf{b}, \mathbf{a})(\tau)=0, \text { for all }|\tau| \in \mathcal{T}_{2} .
\end{aligned}
$$

From Definition 2, C1 shows that each CZCP needs to have two zero autocorrelation zones (ZACZs) when its AAC sums are considered. In this paper, we call them "front-end ZACZ" and "tail-end ZACZ" for time-shifts over $\mathcal{T}_{1}$ and $\mathcal{T}_{2}$, respectively. On the other hand, $\mathrm{C} 2$ shows that each $\mathrm{CZCP}$ needs to have "tail-end zero crosscorrelation zone (ZCCZ)" when its ACC sums are considered. We illustrate the correlation properties of $(N, Z)$-CZCP in Fig. 1. An example of quaternary $(9,3)$-CZCP is given below.

Example 1: Consider the length-9 quaternary pair $(\mathbf{a}, \mathbf{b})$ below.

$$
\mathbf{a}=\omega_{4}^{[0,1,1,2,0,2,1,1,3]}, \mathbf{b}=\omega_{4}^{[0,1,1,0,1,0,3,3,1]} .
$$

$(\mathbf{a}, \mathbf{b})$ is a $(9,3)$-CCP because

$$
\begin{aligned}
& (|\rho(\mathbf{a})(\tau)+\rho(\mathbf{b})(\tau)|)_{\tau=0}^{8} \\
= & \left(18, \mathbf{0}_{1 \times 3}, 2 \sqrt{2}, 2, \mathbf{0}_{1 \times 3}\right), \\
& (|\rho(\mathbf{a}, \mathbf{b})(\tau)+\rho(\mathbf{b}, \mathbf{a})(\tau)|)_{\tau=0}^{8} \\
= & \left(4,4 \sqrt{2}, 2 \sqrt{2}, 2 \sqrt{2}, 4,2, \mathbf{0}_{1 \times 3}\right) .
\end{aligned}
$$


Three main properties of CZCPs are presented below ${ }^{2}$. $\mathrm{P} 1$ : Every $q$-ary $(N, Z)$-CZCP $(\mathbf{a}, \mathbf{b})$ is equivalent to $(N, Z)$ CZCP $(\mathbf{c}, \mathbf{d})$ by dividing $\mathbf{a}$ by $a_{0}$ and $\mathbf{b}$ by $b_{0}$, respectively, i.e., $\mathbf{c}=\mathbf{a} / a_{0}, \mathbf{d}=\mathbf{b} / b_{0}$, where the latter CZCP satisfies

$c_{i}=d_{i}, c_{N-1-i}=-d_{N-1-i}$, for all $i \in\{0,1, \cdots, Z-1\}$.

By utilizing (7), one can readily show that $Z \leq N / 2$.

P2: If $(\mathbf{a}, \mathbf{b})$ is an $(N, Z)-\mathrm{CZCP}$, so are $\left(c_{1} \mathbf{b}, c_{2} \mathbf{a}\right),\left(c_{1} \underline{\mathbf{b}}, c_{2} \underline{\mathbf{a}}\right)$, $\left(c_{1} \underline{\mathbf{b}^{*}}, c_{2} \underline{\mathbf{a}^{*}}\right)$, where $c_{1}, c_{2} \in \mathcal{A}_{q}$. Moreover, if (7) is satisfied by $(\mathbf{a}, \mathbf{b})$, i.e., $a_{i}=b_{i}, a_{N-1-i}=-b_{N-1-i}$, for all $i \in$ $\{0,1, \cdots, Z-1\}$, we have

$$
\begin{aligned}
& \rho\left(\mathbf{a}, \underline{\mathbf{b}^{*}}\right)(\tau)+\rho\left(\mathbf{b},-\underline{\mathbf{a}^{*}}\right)(\tau)=0, \text { for all } \tau . \\
& \rho\left(\mathbf{b}, \underline{\mathbf{b}^{*}}\right)(\tau)+\rho\left(\mathbf{a},-\underline{\mathbf{a}^{*}}\right)(\tau)=0, \text { for all }|\tau| \in \mathcal{T}_{2} .
\end{aligned}
$$

P3: A binary $(N, Z)$-CZCP $(\mathbf{a}, \mathbf{b})$ over $\{-1,1\}$ should have even sequence length $N$ and satisfy the following equation.

$a_{i}+a_{N-1-i}+b_{i}+b_{N-1-i}= \pm 2$, for all $i \in\{0,1, \cdots, Z-1\}$.

Definition 3: By P1, every $q$-ary $\operatorname{CZCP}(\mathbf{a}, \mathbf{b})$ is called perfect if $Z=N / 2$ ( $N$ even). In this case, a perfect $(N, N / 2)$ $\mathrm{CZCP}$ reduces to a sequence pair, called strengthened $G C P$, whose equivalent CZCP (c, d) (see P1) is given in (10).

Note that the even value constraint on sequence length $N$ in P3 may not be necessary for $q$-ary CZCPs with $q>2$. In other words, there exist $q$-ary $(N, Z)$-CZCPs with odd $N$ provided that $Z \leq N / 2$.

Taking advantage of Properties 1-3, we have carried out a computer search for binary (equivalent) $(N, Z)$-CZCPs of lengths up to 26. These search results are presented in Table I of the full paper (see https://arxiv.org/abs/1909.10206.), in which the maximum $Z$ is achieved for every binary CZCP with length $N$. One can see that not all the even $N$ have perfect binary CZCPs with $Z=N / 2$.

Next, we present a systematic approach to construct perfect CZCPs through strengthened GCPs (see Definition 3).

Construction 1: Let $(\mathbf{e}, \mathbf{f})$ be $q$-ary GCP of length $N / 2$. Then, every sequence pair (arranged in matrix form with two rows) in (11) is a perfect $\mathrm{CZCP}$ (i.e., strengthened $G C P$ ). where $v_{1}, v_{2}, v \in \mathbb{Z}_{q}(q$ even $)$ and $v_{1}-v_{2} \in\{0, q / 2\}(\bmod q)$.

Example 2: Consider the length-11 quaternary $\operatorname{GCP}(\mathbf{e}, \mathbf{f})$ below

$$
\mathbf{e}=\omega_{4}^{[0,1,2,0,2,1,3,2,1,1,0]}, \mathbf{f}=\omega_{4}^{[0,0,3,3,3,0,0,1,2,0,2]} .
$$

Let $d=1$. We obtain a perfect CZCP (i.e., strengthened GCP) $(\mathbf{a}, \mathbf{b})$ as follows.

$$
\begin{array}{ll}
\mathbf{a}=\left[\mathbf{e}, \omega_{4}^{d} \cdot \mathbf{f}\right] & =\omega_{4}^{[0,1,2,0,2,1,3,2,1,1,0,1,1,0,0,0,1,1,2,3,1,3]} \\
\mathbf{b}=\left[\mathbf{e},-\omega_{4}^{d} \cdot \mathbf{f}\right] & =\omega_{4}^{[0,1,2,0,2,1,3,2,1,1,0,3,3,2,2,2,3,3,0,1,3,1]}
\end{array}
$$

\footnotetext{
${ }^{2}$ Their proofs can be found in the Appendix part of the full paper whose link is shown in the bottom of the first page.
}

From (12), we have

$$
\begin{aligned}
& (|\rho(\mathbf{a})(\tau)+\rho(\mathbf{b})(\tau)|)_{\tau=0}^{21} \\
= & \left(44, \mathbf{0}_{1 \times 21}\right), \\
& (|\rho(\mathbf{b}, \mathbf{a})(\tau)+\rho(\mathbf{a}, \mathbf{b})(\tau)|)_{\tau=0}^{21} \\
= & \left(0,8 \sqrt{2}, 4,4 \sqrt{2}, 4,0,4,4 \sqrt{2}, 4,0,4, \mathbf{0}_{1 \times 11}\right) .
\end{aligned}
$$

\section{Proposed SM Training Sequences: Design and NUMERICAL EVALUATION}

In this section, we design SM training sequences and evaluate their channel estimation performances over frequencyselective channels with least square estimators. Let $N_{t}$ be the number of TAs. We assume there are four TAs (i.e., $N_{t}=4$ ) and one receive antenna. In the sequel, we illustrate the proposed SM training matrices with the aid of CZCPs.

\section{A. Design of SM Training Sequences}

We employ the perfect $(N=16, Z=8)-\mathrm{CZCP}(\mathbf{a}, \mathbf{b})$ below:

$$
\left[\begin{array}{l}
\mathbf{a} \\
\mathbf{b}
\end{array}\right]=\left[\begin{array}{l}
+++-++-++-+++--- \\
+++-++-+-+---+++
\end{array}\right] .
$$

Based on this perfect CZCP, we generate an SM training matrix with the structure as follows:

$$
\boldsymbol{\Omega}_{1}=\left[\begin{array}{llll:llll}
\mathbf{a} & \mathbf{0} & \mathbf{0} & \mathbf{0} & \mathbf{b} & \mathbf{0} & \mathbf{0} & \mathbf{0} \\
\mathbf{0} & \mathbf{a} & \mathbf{0} & \mathbf{0} & \mathbf{0} & \mathbf{b} & \mathbf{0} & \mathbf{0} \\
\mathbf{0} & \mathbf{0} & \mathbf{a} & \mathbf{0} & \mathbf{0} & \mathbf{0} & \mathbf{b} & \mathbf{0} \\
\mathbf{0} & \mathbf{0} & \mathbf{0} & \mathbf{a} & \mathbf{0} & \mathbf{0} & \mathbf{0} & \mathbf{b}
\end{array}\right]
$$

where $\mathbf{0}$ in $\Omega_{1}$ denotes a row vector consisting of 16 zeros. Each row vector in $\Omega_{1}$ will be sent as a training sequence over a specific transmit antenna. The $(i+1)$-th row vector is simply a right-shifted version of the $i$-row vector for $i=$ $1,2, \cdots, N_{t}-1$. We have the following remarks regarding the design and application of SM training matrices:

Remark 1: In SM system, only one TA is activated over every time-slot, hence SM training matrix (for example, $\boldsymbol{\Omega}_{1}$ ) should be a sparse matrix in which each column has one non-zero entry only. This implies that each training sequence length- $L \boldsymbol{x}_{n}\left(1 \leq n \leq N_{t}\right)$ has $Q \equiv L / N_{t}$ non-zero entries and $\left(N_{t}-1\right) Q$ zeros.

Remark 2: To deal with intersymbol interference (ISI) in frequency-selective channel, a $\mathrm{CP}$ which has duration of not less than the maximum propagation delay should be inserted before each SM training sequence. Note that CP is widely used in orthogonal frequency-division multiplexing (OFDM) and single-carrier frequency-domain equalization (SC-FDE) systems for ISI suppression.

Remark 3: With regard to a training matrix with structure of $\Omega_{1}$ in (15), the front-end ZACZ and the tail-end ZACZ (shown in Fig. 1) of CZCP are helpful in mitigating the ISI of each training sequence and the inter-antenna interference (IAI) between the $i$-th and the $(i+1)$-th TAs $\left(i=1,2, \cdots, N_{t}-1\right)$, respectively. Furthermore, the tail-end ZCCZ of CZCP will help eliminate the IAI between the 1-th and the $N_{t}$-th TAs. 


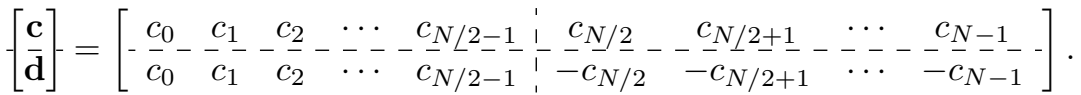



We aim to compare the channel estimation MSE performance with SM training matrices from the length-16 GCP $(\mathbf{c}, \mathbf{d})$ [which is not a CZCP but with the same training structure as that of $\Omega_{1}$ in (15)]

$$
\left[\begin{array}{l}
\mathbf{c} \\
\mathbf{d}
\end{array}\right]=\left[\begin{array}{l}
+++++--+++--+-+- \\
+-+-++--+--+++++
\end{array}\right],
$$

the length-31 m-sequence

$$
\begin{aligned}
\mathbf{a}_{\mathrm{m}}= & {[+---+--+-++--+} \\
& ++++---++-+++-+-],
\end{aligned}
$$

one length-32 CAN sequence ${ }^{3}$ with low aperiodic autocorrelations, the length-13 Barker sequence

$$
\mathbf{a}_{\mathrm{B}}=[+++++--++-+-+],
$$

four length-31 Gold sequences with low periodic crosscorrelations, four length-32 Zadoff-Chu sequences with low periodic cross-correlations, and four "random-on-the-fly" binary sequences of length-32. For the m-sequence, Gold sequences, Zadoff-Chu sequences, CAN sequence and random sequences, we adopt the training matrix structure in (17).

$$
\boldsymbol{\Omega}_{2}=\left[\begin{array}{cccc}
\mathbf{a}_{1} & \mathbf{0} & \mathbf{0} & \mathbf{0} \\
\mathbf{0} & \mathbf{a}_{2} & \mathbf{0} & \mathbf{0} \\
\mathbf{0} & \mathbf{0} & \mathbf{a}_{3} & \mathbf{0} \\
\mathbf{0} & \mathbf{0} & \mathbf{0} & \mathbf{a}_{4}
\end{array}\right]
$$

where $\mathbf{a}_{1}, \mathbf{a}_{2}, \mathbf{a}_{3}, \mathbf{a}_{4}, \mathbf{0}$ are assumed to have identical length. In particular, when a single sequence (i.e., m-sequence or CAN sequence) is used as the "seed", we set $\mathbf{a}_{1}=\mathbf{a}_{2}=\mathbf{a}_{3}=$ $\mathbf{a}_{4}$ in (17). For the length-13 Barker sequence, we adopt the following training matrix

$$
\boldsymbol{\Omega}_{3}=\left[\begin{array}{cccc:cccc}
\mathbf{a}_{\mathrm{B}} & \mathbf{0} & \mathbf{0} & \mathbf{0} & \mathbf{a}_{\mathrm{B}} & \mathbf{0} & \mathbf{0} & \mathbf{0} \\
\mathbf{0} & \mathbf{a}_{\mathrm{B}} & \mathbf{0} & \mathbf{0} & \mathbf{0} & \mathbf{a}_{\mathrm{B}} & \mathbf{0} & \mathbf{0} \\
\mathbf{0} & \mathbf{0} & \mathbf{a}_{\mathrm{B}} & \mathbf{0} & \mathbf{0} & \mathbf{0} & \mathbf{a}_{\mathrm{B}} & \mathbf{0} \\
\mathbf{0} & \mathbf{0} & \mathbf{0} & \mathbf{a}_{\mathrm{B}} & \mathbf{0} & \mathbf{0} & \mathbf{0} & \mathbf{a}_{\mathrm{B}}
\end{array}\right]_{4 \times 104}
$$

For fair comparison, we normalize the energies of all the training sequences to $E=32$.

\section{B. Numerical Evaluation of SM Training Sequences}

For each transmit-receive antenna pair, we consider a $(\lambda+1)$-path channel (separated by integer symbol durations) having uniform power delay profile as follows:

$$
h[t]=\sum_{n=0}^{\lambda} h_{i} \delta[t-n T],
$$

\footnotetext{
${ }^{3} \mathrm{CAN}$ sequences are obtained by minimizing a quadratic function associated with the integrated sidelobe level (ISL) of sequences [25]. With the aid of fast Fourier transform (FFT), CAN sequences can be generated for lengths up to $10^{6}$ or even longer.
}

where $h_{i}$ 's are complex-valued Gaussian random variables with zero mean and $\mathbb{E}\left(\left|h_{i}\right|^{2}\right)=1$. Fig. 2 compares the channel estimation MSEs for different values of multi-paths at EbNo of $16 \mathrm{~dB}$. Note that in Fig. 2, $J$ denotes the number of diagonal blocks in each training matrix. Specifically, $\boldsymbol{\Omega}_{1}, \boldsymbol{\Omega}_{2}, \boldsymbol{\Omega}_{3}$ have $J$ values of $2,1,2$, respectively. One can see that when the number of multi-paths is 9 (or less), our proposed SM training matrix achieves the minimum MSEs. This is because $\mathbf{X}^{\mathrm{H}} \mathbf{X}$, where $\mathbf{X}$ is an enlarged matrix transformed from the training matrix, is an identity matrix multiplied by the training matrix energy which is essential for ensuring the minimum MSEs. More details on the role of $\mathbf{X}^{\mathrm{H}} \mathbf{X}$ can be found in our full paper whose link is given in the bottom of the first page. The same are, however, not attained by training matrices from other seed sequences. As a matter of fact, their channel estimation MSEs display certain distances to the minimum MSE regardless the number of multi-paths. It is interesting to note that the second best channel estimation performance is achieved by the SM training matrix from the length-32 CAN sequence (even better than that from the length-13 Barker sequence) whose aperiodic ISL is minimized. The worst channel estimation performance is achieved by the SM training matrices from random sequences, whereas the second worst channel estimation performance is achieved by the SM training matrices from Gold sequences. Moreover, the channel estimation MSEs corresponding to our proposed SM training matrix start to deviate from, but very close to, the minimum MSE when the number of multi-paths increases to beyond 9. In summary, our proposed SM training matrices give rise to the minimum channel estimation MSEs provided that the number of multi-paths is no greater than $Z+1$; when greater than $Z+1$, our proposed SM training matrices may still give rise to good channel estimation MSEs in the condition that 1) $\mathbf{X}^{\mathrm{H}} \mathbf{X}$ associated to the SM training matrix has full rank and 2) the off-diagonal elements of $\mathbf{X}^{\mathrm{H}} \mathbf{X}$ have small magnitudes.

\section{Conclusions}

This paper is dedicated to the optimal training design in broadband SM systems. We have proposed a new class of sequence pairs called CZCPs in which every CZCP displays zero (symmetrical) AAC sums and zero ACC sums for certain time-shifts. By investigating the structural properties of CZCPs, we have found that perfect CZCPs are equivalent to a subset of GCPs whose first halves are identical and second halves have opposite polarities (See P2 of Section III). Based on this finding, we have shown by systematic constructions that perfect binary CZCPs exist for lengths $2^{\alpha+1} 10^{\beta} 26^{\gamma}$, where $\alpha, \beta, \gamma$ are non-negative integers. By employing CZCP, 


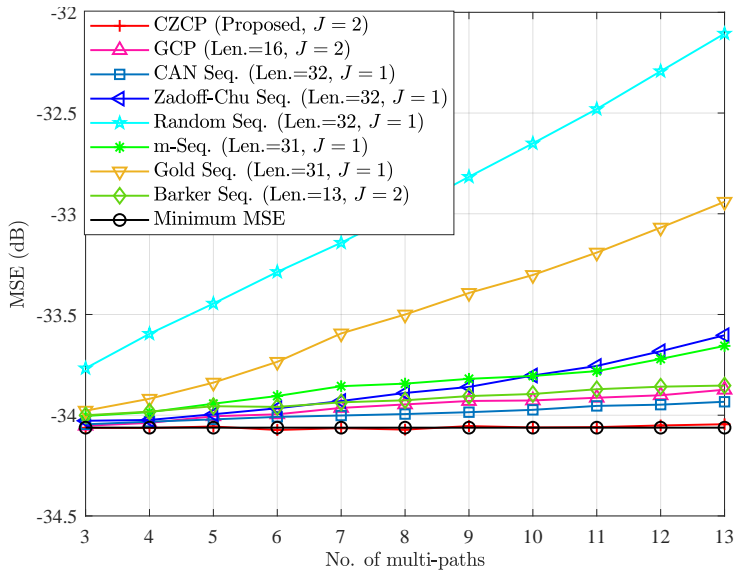

Fig. 2: MSE comparison with training matrices from other sequences for $\mathrm{EbNo}=16 \mathrm{~dB}$.

we have introduced a systematic framework in (15) for the design of optimal SM training matrix. We have presented considerations associated to the design and application of SM training matrices in relation to CZCPs in Remarks 1-3. We have shown via numerical evaluation that our designed SM training matrices lead to minimum channel estimation MSE in quasi-static frequency-selective channels.

Future Works: More systematic constructions (e.g., recursive expansion algorithms) for CZCPs (perfect or non-perfect) may be designed. When a perfect $\mathrm{CZCP}$ is not available, it will be interesting to know what are the almost-perfect CZCPs with respect to different sequence lengths and alphabet sizes. Efficient training design for generalized SM [26] in correlated dispersive channels [27] is also an interesting and challenging issue to be explored.

\section{ACKNOWLEDGMENT}

The authors are deeply indebted to Prof. Pingzhi Fan at Southwest Jiaotong University for suggesting the terminology of "CZCP".

\section{REFERENCES}

[1] R. Mesleh, H. Haas, Y. Lee, and S. Yun, "Interchannel interference avoidance in MIMO transmission by exploiting spatial information," in Proc. 16th IEEE Int. Symp. PIMRC, Berlin, Germany, 2005, vol. 1, pp. 141-145.

[2] M. Wen, B. Zheng, K. Kim, M. Renzo, T. Tsiftsis, K.-C. Chen, and N. Al-Dhahir, "A survey on spatial modulation in emerging wireless systems: research progresses and applications," IEEE J. Sel. Areas Commun., vol. 37, no. 9, pp. 1949-1972, Sep. 2019.

[3] P. Yang, Y. Xiao, Y. L. Guan, K. V. S. Hari, A. Chockalingam, S. Sugiura, H. Hass, M. D. Renzo, C. Masouros, Z. Liu, L. Xiao, S. Li, and L. Hanzo, "Single-carrier SM-MIMO: a promising design for broadband large-scale antenna systems," IEEE Commun. Surveys Tuts., vol. 18, no. 3, pp. 1687 1716, Third Quart., 2016.

[4] J. Jeganathan, A. Ghrayeb, and L. Szczecinski, "Spatial modulation: Optimal detection and performance analysis," IEEE Commun. Lett., vol. 12 , no. 8 , pp. 545-547, Aug. 2008.

[5] M. D. Renzo and H. Haas, "Bit error probability of SM-MIMO over generalized fading channels," IEEE Trans. Veh. Technol., vol. 61, no. 3 , pp. 1124-1144, Mar. 2012.

[6] E. Basar, U. Aygolu, E. Panayirci, and H. Poor, "Performance of spatial modulation in the presence of channel estimation errors," IEEE Commun. Lett., vol. 16, no. 2, pp. 176-179, Feb. 2012.
[7] M. Di Renzo, D. De Leonardis, F. Graziosi, and H. Haas, "Space shift keying (SSK-) MIMO with practical channel estimates," IEEE Trans. Commun., vol. 60, no. 4, pp. 998-1012, Apr. 2012.

[8] S. Sugiura and L. Hanzo, "Effects of channel estimation on spatial modulation," IEEE Signal Process. Lett., vol. 19, no. 12, pp. 805-808, Dec. 2012.

[9] X. Wu, H. Claussen, M. Renzo, and H. Hass, "Channel estimation for spatial modulation," IEEE Trans. Commun., vol. 62 , no. 12 , pp. 4362 4372, Dec. 2014.

[10] P. Fan and W. H. Mow, "On optimal training sequence design for multiple-antenna systems over dispersive fading channels and its extensions," IEEE Trans. Veh. Technol., vol. 53, no. 5, pp. 1623-1626, Sep. 2004.

[11] M. J. E. Golay, "Statatic multislit spectroscopy and its application to the panoramic display of infrared spectra," J. Opt. Soc. Amer., vol. 41, pp. 468-472, 1951.

[12] M. J. E. Golay, "Complementary series," IRE Trans. Inf. Theory, vol. IT-7, pp. 82-87, Apr. 1961.

[13] S. Budišin, "New complementary pairs of sequences," Electron. Lett., vol. 26 , no. 13 , pp. 881-883, Jun. 1990.

[14] S. Budišin, "New multilevel complementary pairs of sequences," Electron. Lett., vol. 26, no. 22, pp. 1861-1863, Oct. 1990.

[15] S. Budišin and P. Spasojevíc, "Paraunitary generation/correlation of QAM complementary sequence pairs," in Proc. Cryptography Commun., vol. 6, no. 1, pp. 59-102, Oct. 2014.

[16] Z. Wang, G. Wu, and D. Ma, "A new method to construct Golay complementary set by paraunitary matrices and Hadamard matrices," in Proc. 9th International Conference on Sequences and Their Applications (SETA-2016), Sep. 2016, pp. 1-12.

[17] S. Das, S. Budišin, S. Majhi, Z. Liu, and Y. L. Guan, "A multiplier-free generator for polyphase complete complementary codes," IEEE Trans. Signal Process., vol. 66, no. 5, pp. 1184-1192, Mar. 2018.

[18] S. Budišin and P. Spasojevíc, "Paraunitary-based Boolean generator for QAM complementary sequences of length $2^{K}$," IEEE Trans. Inf. Theory, vol. 64 , no. 8, pp. 5938-5956, Aug. 2018

[19] J. A. Davis and J. Jedwab, "Peak-to-mean power control in OFDM, Golay complementary sequences, and Reed-Muller codes," IEEE Trans. Inf. Theory, vol. 45, no. 7, pp. 2397-2417, Nov. 1999.

[20] Y. Li, "A construction of general QAM Golay complementary sequences," IEEE Trans. Inf. Theory, vol. 56, no. 11, pp. 5765-5771, Nov. 2010.

[21] Z. Liu, Y. Li, and Y. L. Guan, "New constructions of general QAM Golay complementary sequences," IEEE Trans. Inf. Theory, vol. 59, no. 11, pp. 7684-7692, Nov. 2013.

[22] P. Fan and M. Darnell, Sequence Design for Communications Applications. New York: Wiley, 1996.

[23] Z. Liu, U. Parampalli, and Y. L. Guan, "Optimal odd-length binary Zcomplementary pairs," IEEE Trans. Inf. Theory, vol. 60, no. 9, pp. 57685781, July 2014.

[24] P. Fan, W. Yuan, and Y. Tu, "Z-complementary binary sequences," IEEE Signal Process. Lett., vol. 14, pp. 401-404, Aug. 2007.

[25] P. Stoica, H. He, and J. Li, "New algorithms for designing unimodular sequences with good correlation properties," IEEE Trans. Signal Process., vol. 57, no. 4, pp. 1415-1425, Apr. 2009.

[26] J. Fu, C. Hou, W. Xiang, L. Yan, and Y. Hou, "Generalised spatial modulation with multiple active transmit antennas," in Proc. IEEE GLOBECOM Workshops, Dec. 2010, pp. 839-844.

[27] N. Shariati, J. Wang and M. Bengtsson,"Robust training sequence design for correlated MIMO channel estimation", IEEE Trans. Signal Process., vol. 62, no. 1, pp. 107-120, Jan. 2014. 